PROCEEDINGS OF THE

AMERICAN MATHEMATICAL SOCIETY

Volume 125, Number 6, June 1997, Pages 1801-1806

S 0002-9939(97)04078-1

\title{
RIGIDITY OF COMPACT MANIFOLDS WITH BOUNDARY AND NONNEGATIVE RICCI CURVATURE
}

\author{
CHANGYU XIA
}

(Communicated by Christopher Croke)

\begin{abstract}
Let $\bar{\Omega}$ be an $(n+1)$-dimensional compact Riemannian manifold with nonnegative Ricci curvature and nonempty boundary $M=\partial \bar{\Omega}$. Assume that the principal curvatures of $M$ are bounded from below by a positive constant $c$. In this paper, we prove that the first nonzero eigenvalue $\lambda_{1}$ of the Laplacian of $M$ acting on functions on $M$ satisfies $\lambda_{1} \geq n c^{2}$ with equality holding if and only if $\Omega$ is isometric to an $(n+1)$-dimensional Euclidean ball of radius $\frac{1}{c}$. Some related rigidity theorems for $\bar{\Omega}$ are also proved.
\end{abstract}

\section{INTRODUCTION}

In [SS], Schroeder and Strake proved the following rigidity theorem for manifolds with nonnegative Ricci curvature and convex boundary [SS, Theorem 1]:

Let $\bar{\Omega}$ be a compact Riemannian manifold with convex boundary and nonnegative Ricci curvature. Assume that the sectional curvature is identically zero in some neighborhood $U$ of $\partial \bar{\Omega}$ and that one of the following conditions holds:

a) $\partial \bar{\Omega}$ is simply connected.

b) $\operatorname{dim} \partial \bar{\Omega}$ is even and $\partial \bar{\Omega}$ is strictly convex in some point $p \in \partial \bar{\Omega}$, then $\bar{\Omega}$ is flat.

This theorem is a counterpart for manifolds with nonnegative Ricci curvature of the rigidity results for sectional curvature of Gromov [BGS, §5] which were generalized in [SZ].

As pointed out in [SS], the condition that the metric is flat in a whole neighborhood of $\partial \bar{\Omega}$ in the above result is very strong. Schroeder and Strake asked whether if it suffices to assume that the sectional curvature vanishes only on the boundary. Furthermore, they proved that this is true in the special case of a metric ball (cf. [SS, Theorem 2]).

In this paper, we study a similar rigidity problem for compact manifolds with convex boundary and nonnegative Ricci curvature. By using a different method we prove the following

Theorem 1. Let $\bar{\Omega}$ be an $(n+1)$-dimensional compact Riemannian manifold with nonnegative Ricci curvature and nonempty boundary $M=\partial \bar{\Omega}$ endowed with the metric induced from $\bar{\Omega}$, and let $N$ be the inner unit normal on $M$. Assume that the

Received by the editors December 7, 1995.

1991 Mathematics Subject Classification. Primary 53C20, 53C42.

Key words and phrases. Rigidity, manifolds, Ricci curvature.

This work was supported by the Natural Science Foundation of China, TIT and CNPq.

(C)1997 American Mathematical Society 
principal curvatures of $M$ are bounded from below by a positive constant $c$ (i.e., if we denote by $\sigma$ the second fundamental form of $M$ with respect to $N$, then $\sigma \geq c I$ in the matrix sense). Then, the first nonzero eigenvalue of the Laplacian acting on functions on $M$ satisfies $\lambda_{1}(M) \geq n c^{2}$ with equality holding if and only if $\bar{\Omega}$ is isometric to an $(n+1)$-dimensional Euclidean ball of radius $\frac{1}{c}$.

By using Theorem 1, we can prove the following rigidity theorem which gives a partial answer to the problem proposed by Schroeder and Strake as above.

Theorem 2. Let $\bar{\Omega}$ be an $(n+1)$-dimensional compact Riemannian manifold with nonnegative Ricci curvature and nonempty boundary $M=\partial \bar{\Omega}$. Assume that the sectional curvature $\bar{K}$ of $\bar{\Omega}$ satisfies $\bar{K}(\pi)=0$ for all 2-planes with footpoints on $M$ which are tangent to $M$ and that the mean curvature of $M$ is nonnegative at one point $x \in M$. If $M$ is isometric to a Euclidean $n$-sphere of radius $r$, then $\bar{\Omega}$ is isometric to an $(n+1)$-dimensional Euclidean ball of radius $r$.

By using similar methods as in the proof of Theorem 1, we obtain also the following

Theorem 3. Let $\bar{\Omega}$ be an $(n+1)$-dimensional compact Riemannian manifold with nonnegative Ricci curvature and nonempty boundary $M=\partial \bar{\Omega}$. Assume that the principal curvatures of $M$ are bounded from below by a positive constant $c$ and that the mean curvature of $M$ is bounded from below by $\frac{\lambda_{1}(M)}{n c}$. Then, $\bar{\Omega}$ is isometric to an $(n+1)$-dimensional Euclidean ball of radius $\frac{1}{c}$.

\section{Preliminaries}

In this section, we list some known facts which we need. Let $\bar{\Omega}$ be an $(n+1)$ dimensional compact Riemannian manifold with boundary $M=\partial \bar{\Omega}$. We denote by $\langle$,$\rangle the metric on \bar{\Omega}$ as well as that induced on $M$, and by $\bar{\nabla}$ and $\nabla$ the Riemannian connections on $\bar{\Omega}$ and $M$, respectively. Let $d V$ and $d A$ be the canonical measures on $\bar{\Omega}$ and $M$, respectively, and $V$ and $A$ the volume of $\bar{\Omega}$ and the area of $M$. Given $f$ in $C^{\infty}(\bar{\Omega})$ we denote $z=\left.f\right|_{M}$ and $u=\left.\frac{\partial f}{\partial N}\right|_{M}$, where $N$ is the inner unit normal on $M$. Reilly's formula $[\mathrm{R}]$ states that

$$
\begin{aligned}
& \int_{\bar{\Omega}}\left\{(\bar{\Delta} f)^{2}-\left|\bar{\nabla}^{2} f\right|^{2}-\operatorname{Ric}(\bar{\nabla} f, \bar{\nabla} f)\right\} d V \\
= & \int_{M}\left\{-2(\Delta z) u+n H u^{2}+\sigma(\nabla z, \nabla z)\right\} d A,
\end{aligned}
$$

where $\bar{\nabla} f, \bar{\Delta} f$ and $\bar{\nabla}^{2} f$ are the gradient, the Laplacian and the Hessian, respectively, of $f$ in $\bar{\Omega}$, Ric is the Ricci curvature of $\bar{\Omega}, \nabla z$ and $\Delta z$ are the gradient and the Laplacian, respectively, of $z$ in $M, \sigma$ and $H$ are the second fundamental form and the mean curvature of $M$ with respect to $N$, respectively, i.e., $\sigma(u, v)=\left\langle\bar{\nabla}_{u} v, N\right\rangle$ for $u, v \in T M$ and $H=\frac{1}{n} \operatorname{tr} \sigma$. The principal curvatures of $M$ are defined to be the eigenvalues of $\sigma$. Thus if all the principal curvatures of $M$ are bounded from below by a constant $c$, then $\sigma \geq c I$ in the matrix sense, and the converse is also true.

The following Lemma is an easy consequence of Theorem 1 in [Ro1].

Lemma 1. Let $\bar{\Omega}$ be an $(n+1)$-dimensional compact Riemannian manifold with smooth boundary $M=\partial \bar{\Omega}$ and nonnegative Ricci curvature. If the mean curvature of $M$ satisfies $H \geq \frac{1}{n+1} \cdot \frac{A(M)}{V(\bar{\Omega})}$, then $\bar{\Omega}$ is isometric to a Euclidean ball. Here $A(M)$ and $V(\bar{\Omega})$ denote the area of $M$ and the volume of $\bar{\Omega}$, respectively. 


\section{Proofs of Theorems 1,2 And 3}

Proof of Theorem 1. Let $z$ be an eigenfunction corresponding to the first nonzero eigenvalue $\lambda_{1}$ of the Laplacian of $M$ :

$$
\Delta z+\lambda_{1} z=0 .
$$

Let $f$ in $C^{\infty}(\bar{\Omega})$ be the solution of the Dirichlet problem

$$
\begin{cases}\bar{\Delta} f=0 & \text { in } \bar{\Omega}, \\ f=z & \text { on } M .\end{cases}
$$

It then follows from (1) and the nonnegativity of the Ricci curvature of $\bar{\Omega}$ that

$$
\begin{aligned}
0 & \geq \int_{\bar{\Omega}}\left\{(\bar{\Delta} f)^{2}-\left|\bar{\nabla}^{2} f\right|^{2}-\operatorname{Ric}(\bar{\nabla} f, \bar{\nabla} f)\right\} d V \\
& =\int_{M}\left\{-2(\Delta z) u+n H u^{2}+\sigma(\nabla z, \nabla z)\right\} d A .
\end{aligned}
$$

Since $\sigma \geq \mathrm{c} I$, we have

$$
H \geq c \text { and } \sigma(\nabla z, \nabla z) \geq c|\nabla z|^{2} .
$$

Substituting (2) and (4) into (3) and noticing

$$
\int_{M}|\nabla z|^{2} d A=-\int_{M}(\Delta z) z d A=\lambda_{1} \int_{M} z^{2} d A,
$$

we obtain

$$
\begin{aligned}
0 & \geq \int_{M}\left\{-2\left(-\lambda_{1} z\right) u+n c u^{2}+c|\nabla z|^{2}\right\} d A \\
& =\int_{M}\left\{2 \lambda_{1} z u+n c u^{2}+c \lambda_{1} z^{2}\right\} d A \\
& =\int_{M}\left\{n c\left(u+\frac{\lambda_{1}}{n c} z\right)^{2}+\left(c \lambda_{1}-\frac{\lambda_{1}^{2}}{n c}\right) z^{2}\right\} d A \\
& \geq \int_{M}\left\{\left(c \lambda_{1}-\frac{\lambda_{1}^{2}}{n c}\right) z^{2}\right\} d A .
\end{aligned}
$$

Thus we have from (6)

$$
c \lambda_{1}-\frac{\lambda_{1}^{2}}{n c} \leq 0
$$

or

$$
\lambda_{1} \geq n c^{2} .
$$

This completes the proof of the first part of Theorem 1 .

If $\bar{\Omega}$ is isometric to an $(n+1)$-dimensional Euclidean ball of radius $\frac{1}{c}$, it is well known that $\lambda_{1}(M)=n c^{2}$. Now we assume conversely that $\lambda_{1}=n c^{2}$. In this case inequalities (3), (4) and (6) must take equality sign. In particular, we have

$$
\bar{\nabla}^{2} f=0, \quad H=c \quad \text { and } \quad u=-\frac{\lambda_{1}}{n c} z=-c z .
$$


From $\bar{\nabla}^{2} f=0$, we know that $|\bar{\nabla} f|^{2}$ is a constant and is not zero since $f$ is not a constant. Without loss of generality, we can assume $|\bar{\nabla} f|^{2}=1$. Thus for any point $p \in M$, we have

$$
1=|\bar{\nabla} f|^{2}(p)=|\nabla z|^{2}(p)+u^{2}(p)
$$

It follows from (8) by integration and $u=-c z$, that

$$
\begin{aligned}
A(M) & =\int_{M}\left\{|\nabla z|^{2}+u^{2}\right\} d A \\
& =\int_{M}\left\{\lambda_{1} z^{2}+u^{2}\right\} d A \\
& =\int_{M}\left\{n c^{2} z^{2}+u^{2}\right\} d A \\
& =\int_{M}\left\{(n+1) u^{2}\right\} d A .
\end{aligned}
$$

On the other hand, from

$$
\frac{1}{2} \bar{\Delta}\left(f^{2}\right)=|\bar{\nabla} f|^{2}+f \bar{\Delta} f=1
$$

and the divergence theorem we have

$$
V(\bar{\Omega})=\int_{\bar{\Omega}} \frac{1}{2} \bar{\Delta}\left(f^{2}\right) d V=-\int_{M} z u d A=\int_{M} \frac{u^{2}}{c} d A .
$$

Combining (9) and (11), we find

$$
H=c=\frac{1}{n+1} \cdot \frac{A(M)}{V(\bar{\Omega})} .
$$

From (12) and Lemma 1 we conclude that $\bar{\Omega}$ is isometric to a Euclidean ball. Since $\lambda_{1}(M)=n c^{2}$, the radius of $\bar{\Omega}$ is easily seen to be $\frac{1}{c}$. This completes the proof of Theorem 1 .

Corollary 1. Let $\bar{\Omega}$ be an $(n+1)$-dimensional compact Riemannian manifold with nonnegative Ricci curvature and nonempty boundary $M=\partial \bar{\Omega}$. Assume that the principal curvatures of $M$ are bounded from below by $\sqrt{\frac{\lambda_{1}(M)}{n}}$. Then $\bar{\Omega}$ is isometric to an $(n+1)$-dimensional Euclidean ball of radius $\sqrt{\frac{n}{\lambda_{1}(M)}}$.

Proof. Since $\lambda_{1}=n \cdot\left(\sqrt{\frac{\lambda_{1}}{n}}\right)^{2}$, Corollary 1 follows from the rigidity part of Theorem 1.

In [YY], Yang and Yau proved that if $\left(M, d s^{2}\right)$ is a compact orientable Riemannian surface of genus $g$ with area $A$, then $\lambda_{1}(M) \leq 8 \pi(g+1) A^{-1}$. Thus, Yang and Yau's theorem together with Theorem 1 immediately implies the following

Corollary 2. Let $\bar{\Omega}$ be a 3-dimensional compact Riemannain manifold with nonnegative Ricci curvature and nonempty orientable boundary $M=\partial \bar{\Omega}$. Denote by $g$ the genus of $M$ and assume that the principal curvatures of $M$ are bounded from below by $c>0$. Then the area of $M$ satisfies $A(M) \leq 4 \pi(g+1) c^{-2}$ and equality holds if and only if $\bar{\Omega}$ is isometric to a 3-dimensional Euclidean ball of radius $\frac{1}{c}$. 
Proof of Theorem 2. For any point $p \in M$, we denote by $\sigma_{1}(p), \ldots, \sigma_{n}(p)$ the principal curvatures corresponding to the unit orthogonal principal directions $e_{1}(p), \ldots$, $e_{n}(p)$ of $M$ at $p$, and by $K$ the sectional curvature of $M$. Since $M$ is isometric to an $n$-sphere of radius $r$, it has constant sectional curvature $\frac{1}{r^{2}}$. By the assumption on the sectional curvature of $\bar{\Omega}$ and the Gauss equation, we have for $1 \leq i \neq j \leq n$,

$$
\begin{aligned}
\frac{1}{r^{2}} & =K\left(e_{i}(p) \wedge e_{j}(p)\right) \\
& =\bar{K}\left(e_{i}(p) \wedge e_{j}(p)\right)+\sigma_{i}(p) \sigma_{j}(p)=\sigma_{i}(p) \sigma_{j}(p),
\end{aligned}
$$

where $e_{i}(p) \wedge e_{j}(p)$ denotes the plane spanned by $e_{i}(p)$ and $e_{j}(p)$.

Since $p$ is arbitrary and the principal curvatures are continuous functions on $M$, we conclude therefore that either $\sigma_{1}=\cdots=\sigma_{n} \equiv \frac{1}{r}$ or $\sigma_{1}=\cdots=\sigma_{n} \equiv-\frac{1}{r}$. The latter possibility is ruled out since the mean curvature of $M$ is nonnegative at the point $x$. On the other hand, since $M$ is isometric to a Euclidean $n$-sphere of radius $r$, it is well known that $\lambda_{1}(M)=n \cdot \frac{1}{r^{2}}$. Hence, $\bar{\Omega}$ is isometric to an $(n+1)$-dimensional Euclidean ball of radius $r$ by Theorem 1 . This completes the proof of Theorem 2 .

Proof of Theorem 3. As in the proof of Theorem 1, let $z$ be an eigenfunction corresponding to the first nonzero eigenvalue $\lambda_{1}$ of the Laplacian of $M$ and let $f$ in $C^{\infty}(\bar{\Omega})$ be the solution of the Dirichlet problem

$$
\begin{cases}\bar{\Delta} f=0 & \text { in } \bar{\Omega} \\ f=z & \text { on }\end{cases}
$$

From (1), the assumption on the Ricci curvature of $\bar{\Omega}$ and

$$
\sigma(\nabla z, \nabla z) \geq c|\nabla z|^{2}, \quad H \geq \frac{\lambda_{1}}{n c},
$$

we have

$$
\begin{aligned}
0 & \geq \int_{\bar{\Omega}}\left\{(\bar{\Delta} f)^{2}-\left|\bar{\nabla}^{2} f\right|^{2}-\operatorname{Ric}(\bar{\nabla} f, \bar{\nabla} f)\right\} d V \\
& =\int_{M}\left\{-2(\Delta z) u+n H u^{2}+\sigma(\nabla z, \nabla z)\right\} d A \\
& \geq \int_{M}\left\{2 \lambda_{1} z u+\frac{\lambda_{1}}{c} u^{2}+c \lambda_{1} z^{2}\right\} d A \\
& =\int_{M} \frac{\lambda_{1}}{c}(u+c z)^{2} d A \\
& \geq 0 .
\end{aligned}
$$

Thus, the inequalities (14) and (15) must take equality sign. In particular, we have

$$
\bar{\nabla}^{2} f=0, \quad H=\frac{\lambda_{1}}{n c} \quad \text { and } \quad u=-c z .
$$


As in the proof of the second part of Theorem 1 we can assume from $\bar{\nabla}^{2} f=0$ that $1=|\bar{\nabla} f|^{2}$ and so $1=|\nabla z|^{2}(p)+u^{2}(p)$ for any $p \in M$. Consequently, we obtain

$$
\begin{aligned}
A(M) & =\int_{M}\left\{|\nabla z|^{2}+u^{2}\right\} d A=\int_{M}\left\{\lambda_{1} z^{2}+u^{2}\right\} d A \\
& =\int_{M}\left\{\frac{\lambda_{1}}{c^{2}}+1\right\} u^{2} d A=\left(\frac{\lambda_{1}}{c^{2}}+1\right) c \cdot \int_{M}(-z u) d A \\
& =\left(\frac{\lambda_{1}}{c^{2}}+1\right) c \cdot \int_{\bar{\Omega}} \frac{1}{2} \bar{\Delta}\left(f^{2}\right) d V=\left(\frac{\lambda_{1}}{c^{2}}+1\right) c V(\bar{\Omega}) .
\end{aligned}
$$

It then follows from $H=\frac{\lambda_{1}}{n c} \geq c$ and (17) that

$$
\frac{A(M)}{V(\bar{\Omega})}=\frac{\lambda_{1}}{c}+c \leq n H+H=(n+1) H,
$$

or

$$
H \geq \frac{A(M)}{(n+1) V(\bar{\Omega})} .
$$

Hence, $\bar{\Omega}$ is isometric to an $(n+1)$-dimensional Euclidean ball by Lemma 1 . If we denote by $r$ the radius of $\bar{\Omega}$, then the principal curvatures of $M$ are all equal to $\frac{1}{r}$, that is, $\sigma=\frac{1}{r} I$. We conclude therefore from $\sigma(\nabla z, \nabla z)=c|\nabla z|^{2}$ that $r=\frac{1}{c}$. This completes the proof of Theorem 3.

\section{REFERENCES}

[BGS] Ballmann, W., Gromov, M. and Schroeder, V., Manifolds of nonpositive curvature, Birkhäuser, Basel-Boston, 1985. MR 87h:53050

[CW] Choi, H. I. and Wang, A. N., A first eigenvalue estimate for minimal hypersurfaces, J. Diff. Geom. 18 (1983), 559-562. MR 85d:53028

[R] Reilly, R., Applications of the Hessian operator in Riemannian manifolds, Indiana Univ. Math. J. 26 (1977), 459-472. MR 57:13799

[Ro1] Ros, A., Compact hypersurfaces with constant higher order mean curvature, Revista matemática Iberroamericana 3 (1987), 447-453. MR 90c:53160

[Ro2] Ros, A., Compact hypersurfaces with constant scalar curvature and a congruence theorem, J. Diff. Geom. 27 (1988), 215-220. MR 89b:53096

[SS] Schroeder, V. and Strake, M., Rigidity of convex domains in manifolds with nonnegative Ricci and sectional curvature, Comment. Math. Helvetici 64 (1989), 173-186. MR 90h:53042

[SZ] Schroeder, V. and Ziller, W., Local rigidity of symmetric spaces, Trans. of the Amer. Math. Soc. 320 (1990), 145-160. MR 90k:53089

[X] Xia, C. Y., Rigidity and sphere theorem for manifolds with positive Ricci curvature, manuscripta math. 85 (1994), 79-87. MR 95j:53057

[YY] Yang, P. and Yau, S. T., Eigenvalues of the Laplacian of compact Riemannian surfaces and minimal submanifolds, Ann. Scuola norm. Sup. Pisa 7 (1980), 55-63. MR 81m:58084

Department of Mathematics, University of Science and Technology of China, Hefei, Anhui 230026, People's Republic of China

Current address: Instituto de Matematica Pure e Aplicada, Estrada Dona Castorina 110, Jardim Botanico 22460-320, Rio de Janeiro, RJ Brasil

E-mail address: xiacy@impa.br 